\title{
Performance of UPFD scheme under some different regimes of advection, diffusion and reaction
}

Appanah Rao Appadu , (Department of Mathematics and Applied Mathematics, University of Pretoria, Pretoria, South Africa)

\begin{abstract}
An unconditionally positive definite finite difference scheme termed as UPFD has been derived in order to approximate a linear advection-diffusion-reaction equation which models exponential travelling waves and the coefficients of advection, diffusion and reactive terms have been chosen as one [6]. In this work, we test UPFD scheme under some other different regimes of advection, diffusion and reaction. We consider the case when the coefficient of advection, diffusion and reaction are all equal to one and also cases under which advection or diffusion or reaction is more important. Some errors such as $L_{1}$ error, dispersion, dissipation errors and relative errors are tabulated. Moreover, we compare some spectral properties of the method under different regimes. We obtain the variation of the following quantities with respect to the phase angle; modulus of exact amplification factor, modulus of amplification factor of the scheme and relative phase error.
\end{abstract}

\section{Keywords:}

advection-diffusion-reaction, finite difference, unconditionally stable, error rate, dispersion, dissipation, relative error.

\section{Introduction}

Advection-diffusion-reaction partial differential equations are among the most widespread in many fields of engineering and science. These applications include the transport of air, adsorption of pollutants in soil, diffusion of neutrons, food processing, modelling of biological systems, modelling of semiconductors, oil reservoir flow transport among others [5]. There are only few cases for which the analytical solution to the advection-diffusion-reaction equations exist, hence the need for numerical techniques for accurate and efficient solution of such equations. Numerical solution to quite complicated partial differential equations such as time-fractional pdes has been studied in $[1,9,10]$.

Stability is an essential concern when studying the convergence of a numerical method approximating a partial differential equation and it is often very difficult to obtain stability limits for a numerical method. In the past, many attempts were made in order to obtain stability of numerical methods discretising convection-diffusion equations. Several techniques from previous works have been reviewed in [18] and the scheme used is the Forward-time and space-centred scheme. We next highlight some previous works on stability of numerical methods discretising advection-diffusion equations.

Fromm [11] applied Von Neumann analysis and obtained stability limits for the two-dimensional vorticity transport equation. The mistake made by Fromm is that he derived sufficient stability conditions that were not necessary conditions. He wrongly assumed that these conditions were both necessary and sufficient. In 1968, Hirt [14] obtained stability conditions after examining the truncation and CFL condition. Such techniques only give necessary conditions. It was proved later that the stationary conditions obtained by Hirt are both necessary and sufficient. Siemieniuch and Gladwell [17] made use of spectral radius for stability and got different conditions from those of Fromm and Hirt. Siemieniuch and Gladwell only obtained necessary conditions for stability. Rigal [15] used Von Neumann analysis to derive stability limits. He obtained correct stability limits and these were proved to be necessary for stability. Clancy [7] obtained the same stability limits as Rigal by using Von Neumann analysis but could only prove that they are sufficient for stability. In 1984, Hindmarsch et al. [12] made use of Von Neumann analysis and proved that the correct limits are necessary and sufficient stability conditions. 
Difficulties can arise in stability analysis. It is important to have a full understanding of whether the conditions obtained for stability are sufficient, necessary or necessary and sufficient. The advectiondiffusion-reaction is quite similar to the advection-diffusion equation, it has an extra reaction term and therefore obtaining stability of some numerical methods discretising advection-diffusion-reaction equation is not easy as is the case with numerical methods discretising advection-diffusion equations. To avoid difficulty involved with obtaining region of stability, we shall consider unconditionally stable finite difference schemes discretising advection-diffusion-reaction equations.

\section{Organisation of paper}

The paper is organised as follows. In section 3, we describe the numerical experiment considered, present the numerical method and obtain expressions for the exact amplification factor, numerical amplification factor and relative phase error and in section 4, we describe briefly the quantification of errors. In sections 5 to 8, we tabulate the errors, present some numerical results from UPFD for some different regimes. Section 9 highlights the salient features of the paper. Coding and plotting of graphs are performed using matlab software.

\section{Numerical Experiment}

We solve an advection-diffusion-reaction equation which models exponential travelling waves [6]. The equation is given by

$$
\frac{\partial u}{\partial t}+a \frac{\partial u}{\partial x}-D \frac{\partial^{2} u}{\partial x^{2}}=-\kappa u,
$$

subject to the following initial and boundary conditions;

$u(x, 0)=\exp (-x), u(0, t)=\exp [(-\kappa+D+a) t]$ and $u_{x}(10, t)=-u(10, t)$ where $0 \leq x \leq 10$ and $0 \leq t \leq 0.85$.

The exact solution is given by

$$
u(x, t)=\exp [(-\kappa+D+a) t-x] .
$$

We solve this problem taking into consideration some different regimes:

(i) case 1: $a=1, D=1, \kappa=1$.

(ii) case 2 where the advective regime is more important: $a=5, D=1, \kappa=1$ and $a=10, D=1$, $\kappa=1$.

(iii) case 3 where the diffusive regime is more important: $a=1, D=5, \kappa=1$ and $a=1, D=10$, $\kappa=1$.

(iv) case 4 where the reactive regime is more important: $a=1, D=1, \kappa=5$.

The Unconditionally Positive Finite Difference (UPFD) method is given by [6]

$$
\frac{u_{i}^{n+1}-u_{i}^{n}}{k}+a\left(\frac{u_{i}^{n+1}-u_{i-1}^{n}}{h}\right)-D\left(\frac{u_{i+1}^{n}-2 u_{i}^{n+1}+u_{i-1}^{n}}{h^{2}}\right)=-\kappa u_{i}^{n+1},
$$

which simplifies as

$$
u_{i}^{n+1}=\frac{u_{i}^{n}+(a \lambda+D \beta) u_{i-1}^{n}+D \beta u_{i+1}^{n}}{1+\kappa k+a \lambda+2 D \beta},
$$

where $\lambda=\frac{k}{h}$ and $\beta=\frac{k}{h^{2}}, h$ is the mesh size and $k$ is the temporal step size.

The scheme is positive definite for all $k, h>0$. Hence, the scheme is unconditionally stable for all $k, h>0$. However, the method is not unconditionally consistent. There are extra truncation error terms due to the fact that in the approximation to the first and second derivatives with respect to $x$, 
there are terms evaluated at different time levels.

There are two ways how the scheme can be used. One way to deal with this situation is to choose the time step size dependent on the mesh size in such a way that the inconsistent terms go to zero as the mesh is refined [6].

Using Taylor expansion about the point $(i, n)$ and assuming that $u$ is continuous and the derivatives of $u$ are bounded, the modified equation of UPFD scheme discretising Eq. (1) is given by

$$
\begin{gathered}
\frac{\partial u}{\partial t}+a \frac{\partial u}{\partial x}-D \frac{\partial^{2} u}{\partial x^{2}}+\kappa u=-\left(\kappa k+\frac{a k}{h}+\frac{2 D k}{h^{2}}\right) \frac{\partial u}{\partial t}-\left(\frac{k}{2}+\frac{\kappa k^{2}}{2}+\frac{a k^{2}}{2 h}+\frac{D k^{2}}{h^{2}}\right) \frac{\partial^{2} u}{\partial t^{2}} \\
-\left(\frac{k^{2}}{6}+\frac{\kappa k^{3}}{6}+\frac{a k^{3}}{6 h}+\frac{D k^{3}}{3 h^{2}}\right) \frac{\partial^{3} u}{\partial t^{3}}+\frac{a h}{2} \frac{\partial^{2} u}{\partial x^{2}}-\frac{a h^{2}}{6} \frac{\partial^{3} u}{\partial x^{3}}+\cdots
\end{gathered}
$$

The second alternative is to modify the scheme by including some terms in order to obtain a consistent approximation. The latter option was used in [6] with spatial mesh size chosen as 0.1 and temporal step size as 0.005. We now show how this approach works. The PDE to solve is given by Eq. (1) and the modified equation is given by Eq. (5). The main term causing inconsistency is $\alpha \frac{\partial u}{\partial t}$ where $\alpha=\frac{k}{h^{2}}(2 D+a h)$. If we add this term to the left hand side of Eq. (1), we get

$$
\frac{\partial u}{\partial t}(1+\alpha)+a \frac{\partial u}{\partial x}-D \frac{\partial^{2} u}{\partial x^{2}}=-\kappa u
$$

Instead of solving Eq (1) using UPFD, Eq. (6) is solved using UPFD as the latter form reduces errors due to inconsistent terms. A new time scale, $T=\frac{t}{1+\alpha}$ is defined. Since $\frac{\partial u}{\partial t}=\frac{\partial u}{\partial T} \times \frac{\partial T}{\partial t}$, we thus get $\frac{\partial T}{\partial t}=\frac{1}{1+\alpha}$. Eq. (6) can be written as

$$
\frac{\partial u}{\partial T}+a \frac{\partial u}{\partial x}-D \frac{\partial^{2} u}{\partial x^{2}}=-\kappa u
$$

Eq. (7) is similar to the original equation but with a new time scale. We solve Eq. (7) using UPFD scheme where $T=\frac{t}{1+\alpha}$ for $t \in[0,0.85]$ and $\alpha=\frac{k}{h^{2}}(2 D+a h)$.

In this work, we use the UPFD method to solve some advection-diffusion-reaction problems using $h=0.1$ and some different values of temporal step size. We use the first alternative.

\section{Amplification factor and Relative Phase Error}

One of the most common ways of measuring the relative merits of a scheme for advection is to consider the scheme's dispersion and dissipation [19]. All linear numerical schemes are either dispersive or dissipative [21]. The amplification factor of the UPFD scheme is given by

$$
\xi_{\text {num }}=\frac{1+(a \lambda+2 D \beta) \cos (w)}{1+\kappa k+a \lambda+2 D \beta}-\frac{a \lambda \sin (w)}{1+\kappa k+a \lambda+2 D \beta} I .
$$

The relative phase error (RPE) is a measure of the dispersive property of a scheme. It is a ratio and measures the velocity of the computed waves to that of the physical waves [22]. If the RPE is greater than one, the computed waves appear to move faster than the physical waves [13]. A ratio less than one implies that the computed waves will move slower than the physical waves, causing phase lag. When $R P E=1$, there is no phase error. The RPE is calculated $\operatorname{as} \frac{\arg \left(\xi_{\text {num }}\right)}{\arg \left(\xi_{\text {exact }}\right)}$ [8]. Using the perturbation, $u=\exp (\alpha t) \exp (I \theta x)[8,16,20]$ to the partial differential equation given by (1) where $\theta$ is the wavenumber and $\alpha$ is the dispersion relation, we obtain $\alpha=-a I \theta-D \theta^{2}-\kappa$. The exact amplification factor, $\xi_{\text {exact }}$ is computed as $\frac{u(x, t+\Delta t)}{u(x, t)}[20]$ and we thus have

$$
\xi_{\text {exact }}=\exp (-a \theta k I) \exp \left[-\left(\kappa+D(w / h)^{2}\right) k\right]
$$


where $w=\theta h$.

Hence the RPE of the UPFD scheme discretising Eq. (1) is given by

$$
-\frac{h}{a k w} \tan ^{-1}\left(\frac{\Im\left(\xi_{\text {num }}\right)}{\Re\left(\xi_{\text {num }}\right)}\right)
$$

In the following section, we describe how the total mean square error can be quantified into dispersion and dissipation errors using the technique of Takacs [19].

\section{Quantification of Errors}

Finite difference schemes used to solve partial differential equations will often lose energy as time progresses, this property is called numerical dissipation. In the case of dispersive schemes, oscillations are generated in regions of discontinuity. We make use of the technique devised by Takacs [19] in order to quantify errors from numerical results into dispersion and dissipation errors. The total mean square error (TMSE) is calculated as $\frac{1}{N} \sum_{i=1}^{N}\left(u_{i}-v_{i}\right)^{2}$, where $u_{i}$ represents the analytical solution and $v_{i}$, the numerical (discrete) solution at a given grid point $i$ and $N$ is the number of discrete points.

The absolute error is given by

$$
E=\sum_{i=1}^{N}\left|u_{i}-v_{i}\right| .
$$

The total mean square error is calculated as

$$
\begin{aligned}
& E_{T M S}=\frac{1}{N} \sum_{i=1}^{N}\left(u_{i}-v_{i}\right)^{2}, \text { or } \\
& E_{T M S}=\frac{1}{N} \sum_{i=1}^{N} u_{i}^{2}-\frac{2}{N} \sum_{i=1}^{N} u_{i} v_{i}+\frac{1}{N} \sum_{i=1}^{N} v_{i}^{2},
\end{aligned}
$$

where $N$ is the number of grid points.

The sample variances for the analytical and numerical solutions are respectively given by

$$
\begin{aligned}
& \delta^{2}(u)=\frac{1}{N} \sum_{i=1}^{N}\left(u_{i}-\bar{u}\right)^{2} \text { and } \\
& \delta^{2}(v)=\frac{1}{N} \sum_{i=1}^{N}\left(v_{i}-\bar{v}\right)^{2} .
\end{aligned}
$$

Expanding the sample variance, we obtain

$$
\begin{aligned}
& \delta^{2}(u)=\frac{1}{N} \sum_{i=1}^{N} u_{i}^{2}-\frac{2}{N} \sum_{i=1}^{N} u \bar{u}+\frac{1}{N} \sum_{i=1}^{N} \bar{u}^{2}, \\
& \delta^{2}(v)=\frac{1}{N} \sum_{i=1}^{N} v_{i}^{2}-\frac{2}{N} \sum_{i=1}^{N} v_{i} \bar{v}+\frac{1}{N} \sum_{i=1}^{N} \bar{v}^{2} .
\end{aligned}
$$

The total mean square error can be expressed as

$$
\begin{aligned}
E_{T M S} & =\frac{1}{N} \sum_{i=1}^{N}\left(u_{i}-\bar{u}\right)^{2}+\frac{1}{N} \sum_{i=1}^{N}\left(v_{i}-\bar{v}\right)^{2}+\frac{2}{N} \sum_{i=1}^{N} u_{i} \bar{u}_{i} \\
& +\frac{2}{N} \sum_{i=1}^{N} v_{i} \bar{v}-\frac{1}{N} \sum_{i=1}^{N}(\bar{u})^{2}-\frac{1}{N} \sum_{i=1}^{N}(\bar{v})^{2}-\frac{2}{N} \sum_{i=1}^{N} u_{i} v_{i} .
\end{aligned}
$$


or

$$
\begin{aligned}
E_{T M S} & =\delta^{2}(u)+\delta^{2}(v)+2\left(\frac{1}{N} \sum_{i=1}^{N} u_{i} \bar{u}\right)+2\left(\frac{1}{N} \sum_{i=1}^{N} v_{i} \bar{v}\right) \\
& -\frac{1}{N} \sum_{i=1}^{N}(\bar{u})^{2}-\frac{1}{N} \sum_{i=1}^{N}(\bar{v})^{2}-2\left(\frac{1}{N} \sum_{i=1}^{N} u_{i} v_{i}-\bar{u} \bar{v}\right)-2 \bar{u} \bar{v}
\end{aligned}
$$

On further simplification, we have

$$
E_{T M S}=\delta^{2}(u)+\delta^{2}(v)+(\bar{u}-\bar{v})^{2}-2 \operatorname{Cov}(u, v) .
$$

The correlation coefficient is defined as

$$
\rho=\frac{\operatorname{Cov}(u, v)}{\delta(u) \delta(v)} .
$$

Hence, the covariance can be expressed as

$$
\operatorname{Cov}(u, v)=\rho \delta(u) \delta(v) .
$$

We thus have,

$$
\begin{aligned}
E_{T M S} & =\delta^{2}(u)+\delta^{2}(v)+(\bar{u}-\bar{v})^{2}-2 \rho \delta(u) \delta(v) \\
& =\delta^{2}(u)+\delta^{2}(v)+(\bar{u}-\bar{v})^{2}-2 \rho \delta(u) \delta(v)+2 \delta(u) \delta(v)-2 \delta(u) \delta(v) \\
& =\delta^{2}(u)+\delta^{2}(v)-2 \delta(u) \delta(v)+(\bar{u}-\bar{v})^{2}-2 \rho \delta(u) \delta(v)+2 \delta(u) \delta(v) \\
& =(\delta(u)-\delta(v))^{2}+(\bar{u}-\bar{v})^{2}+2(1-\rho) \delta(u) \delta(v) .
\end{aligned}
$$

Setting $\rho=1$, we obtain

$$
E_{T M S}=(\delta(u)-\delta(v))^{2}+(\bar{u}-\bar{v})^{2} .
$$

Hence the dispersion error is

$$
2(1-\rho) \delta(u) \delta(v)
$$

while the dissipation error is

$$
(\delta(u)-\delta(v))^{2}+(\bar{u}-\bar{v})^{2} .
$$

The error rate with respect to $L_{1}$ norm is given by

$$
E_{\text {num }}=h \sum_{i=1}^{N}\left|u_{i}-v_{i}\right| .
$$

Some interesting applications of the concept of quantification of errors into dispersion and dissipation can be found in $[2,3,19]$.

\section{Case 1: Coefficient of advection, diffusion and reaction are equal}

We present $L_{1}$ errors, dissipation, dispersion errors for the case $a=1, D=1, \kappa=1$ at some different values of $k$ with $h$ chosen as 0.1 and 0.2 . It is seen that at time $t=0.85$, the errors are much affected by the values of $k$ used; lowest values of $k$ cause errors to be least.

From the values of $k$ used in Table (1), we can see that $k=0.00025$ is the optimal one in terms of 


\begin{tabular}{|c|c|c|c|c|c|c|}
\hline$k$ & $k / h$ & $k /\left(h^{2}\right)$ & $L_{1}$ error & Diss. error & Disp. error & TMSE \\
\hline 0.00025 & 0.0025 & 0.025 & $3.1353 \times 10^{-4}$ & $6.3451 \times 10^{-10}$ & $1.7504 \times 10^{-9}$ & $2.3849 \times 10^{-9}$ \\
0.0005 & 0.005 & 0.05 & $3.2759 \times 10^{-2}$ & $1.7813 \times 10^{-5}$ & $1.2860 \times 10^{-5}$ & $3.0673 \times 10^{-5}$ \\
0.001 & 0.01 & 0.1 & $9.2238 \times 10^{-2}$ & $1.4473 \times 10^{-4}$ & $1.0429 \times 10^{-4}$ & $2.4902 \times 10^{-4}$ \\
0.002 & 0.02 & 0.2 & $1.9334 \times 10^{-1}$ & $6.5967 \times 10^{-4}$ & $4.7452 \times 10^{-4}$ & $1.1342 \times 10^{-3}$ \\
0.005 & 0.05 & 0.5 & $4.0421 \times 10^{-1}$ & $3.1314 \times 10^{-3}$ & $2.2154 \times 10^{-3}$ & $5.3468 \times 10^{-3}$ \\
0.01 & 0.1 & 1 & $6.0366 \times 10^{-1}$ & $7.6423 \times 10^{-3}$ & $5.2050 \times 10^{-3}$ & $1.2847 \times 10^{-2}$ \\
\hline
\end{tabular}

Table 1: Errors obtained from UPFD scheme at some different values of $k$ with $h=0.1, a=1, D=1$ and $\kappa=1$.

\begin{tabular}{|c|c|c|c|c|c|c|}
\hline$k$ & $k / h$ & $k /\left(h^{2}\right)$ & $L_{1}$ error & Diss. error & Disp. error & TMSE \\
\hline 0.00025 & 0.00125 & 0.00625 & $6.1260 \times 10^{-2}$ & $5.6336 \times 10^{-5}$ & $4.7763 \times 10^{-5}$ & $1.0410 \times 10^{-4}$ \\
0.0005 & 0.0025 & 0.0125 & $5.1831 \times 10^{-2}$ & $4.0435 \times 10^{-5}$ & $3.4327 \times 10^{-5}$ & $7.4762 \times 10^{-5}$ \\
0.001 & 0.005 & 0.025 & $3.3428 \times 10^{-2}$ & $1.6905 \times 10^{-5}$ & $1.4389 \times 10^{-5}$ & $3.1294 \times 10^{-5}$ \\
0.002 & 0.01 & 0.05 & $1.6606 \times 10^{-3}$ & $4.2139 \times 10^{-8}$ & $3.6040 \times 10^{-8}$ & $7.8179 \times 10^{-8}$ \\
0.005 & 0.025 & 0.125 & $9.4970 \times 10^{-2}$ & $1.4164 \times 10^{-4}$ & $1.2246 \times 10^{-4}$ & $2.6410 \times 10^{-4}$ \\
0.01 & 0.05 & 0.25 & $2.1970 \times 10^{-1}$ & $7.8823 \times 10^{-4}$ & $6.8923 \times 10^{-4}$ & $1.4770 \times 10^{-3}$ \\
\hline
\end{tabular}

Table 2: Errors obtained from UPFD scheme at some different values of $k$ with $h=0.2, a=1, D=1$ and $\kappa=1$.

dissipation, dispersion errors and $L_{1}$ error. As $k$ is increased, the errors increase. We also note that the dispersion and dissipation errors are almost the same at a given value of $k$. Table (2) displays the errors when $h$ is chosen as 0.2 . As we increase $k$, the error initially decreases and at $k=0.002$, the errors are least but errors increase again as the value of $k$ is increased. Based on Tables (1) and (2), there is no common value for $k / h$ or $k / h^{2}$ for which errors are least. This is in line with observation made in [2]. When the 1-D linear advection equation is approximated by a given numerical method, the plots of the modulus of the amplification factor and relative phase error vs phase angle depend on CFL number (which is equal to $\frac{a k}{h}$ ). However, in the case of 1-D advection-diffusion equation discretised by a finite difference method, the plots of modulus of numerical amplification factor and relative phase error depends on $k, h$ and not on the CFL number.

We present profiles from exact solution, initial solution and numerical solution (at some values of $k$ namely; $k=0.00025, k=0.001, k=0.01)$ and $h=0.1$ in Figs. (1(a)), (1(b)) and (1(c)). All the profiles show that the value of the solution decreases monotonically with increase in $x$. The initial solution is shown by green line while the exact and numerical profiles are denoted by blue and red lines respectively. The peak of the initial solution is at $x=0$ and corresponds to $u=1.0$. The peak of the numerical solution at time $t=0.85$ is approximately 2.3. As expected based on the errors from Table (1), the deviation between numerical and exact profiles increases as the value of $k$ is increased.

To understand better why the numerical results are very much dependent on the temporal step size, we study the variation of the modulus of the exact amplification factor, modulus of numerical amplification factor and relative phase error, all versus the phase angle at different values of $k$ with $h=0.1$. The plots are shown in Figs. (2(a)), (2(b)), (2(c)) and (2(d)). Figs. (2(a)) and (2(b)) show that $\left|\xi_{\text {num }}\right|$ is very close to $\left|\xi_{\text {exact }}\right|$ at lowest values of $k$, with $h$ being 0.1 . For instance at $k=0.01$, there is much deviation between $\left|\xi_{\text {num }}\right|$ and $\left|\xi_{\text {exact }}\right|$. Figs. $(2(\mathrm{c}))$ and $(2(\mathrm{~d}))$ show the plot of RPE vs phase angle at some different values of $k$. For values of $k$ closest to zero, the values of the RPE are close to one especially at low phase angles. We also note that when $w=0$, the RPE is not always equal to one as is expected for consistent finite difference schemes discretising advection-diffusion-reaction equations [4]. We conclude that UPFD does preserve dispersive properties to some extent only at low phase angles and for values of $k$ very close to zero. 


\begin{tabular}{|c|c|c|c|c|}
\hline$x$ & Exact & Numerical & Absolute Error & Relative Error \\
\hline 0.0 & 2.3396 & 2.3396 & 0 & 0 \\
1.0 & $8.6071 \times 10^{-1}$ & $8.6062 \times 10^{-1}$ & $8.8849 \times 10^{-5}$ & $1.0323 \times 10^{-4}$ \\
2.0 & $3.1664 \times 10^{-1}$ & $3.1658 \times 10^{-1}$ & $5.9600 \times 10^{-5}$ & $1.8823 \times 10^{-4}$ \\
3.0 & $1.1648 \times 10^{-1}$ & $1.1646 \times 10^{-1}$ & $2.8180 \times 10^{-5}$ & $2.4192 \times 10^{-4}$ \\
4.0 & $4.2852 \times 10^{-2}$ & $4.2841 \times 10^{-2}$ & $1.1375 \times 10^{-5}$ & $2.6544 \times 10^{-4}$ \\
5.0 & $1.5764 \times 10^{-2}$ & $1.5760 \times 10^{-2}$ & $4.2899 \times 10^{-6}$ & $2.7213 \times 10^{-4}$ \\
6.0 & $5.7990 \times 10^{-3}$ & $5.7980 \times 10^{-3}$ & $1.5851 \times 10^{-6}$ & $2.7332 \times 10^{-4}$ \\
7.0 & $2.1335 \times 10^{-3}$ & $2.1329 \times 10^{-3}$ & $5.8333 \times 10^{-7}$ & $2.7342 \times 10^{-4}$ \\
8.0 & $7.8486 \times 10^{-4}$ & $7.8465 \times 10^{-4}$ & $2.1385 \times 10^{-7}$ & $2.7246 \times 10^{-4}$ \\
9.0 & $2.8874 \times 10^{-4}$ & $2.8866 \times 10^{-4}$ & $7.1437 \times 10^{-8}$ & $2.4741 \times 10^{-4}$ \\
10.0 & $1.0622 \times 10^{-4}$ & $1.0625 \times 10^{-4}$ & $2.9797 \times 10^{-8}$ & $2.8052 \times 10^{-4}$ \\
\hline
\end{tabular}

Table 3: Absolute and relative errors obtained from UPFD scheme using $k=0.00025$ with $h=0.1$, $a=1, D=1$ and $\kappa=1$.

\begin{tabular}{|c|c|c|c|c|}
\hline$x$ & Exact & Numerical & Absolute Error & Relative Error \\
\hline 0.0 & 2.339647 & 2.339647 & 0 & 0 \\
1.0 & 0.860708 & 0.564422 & 0.296286 & 0.344235 \\
2.0 & 0.316637 & 0.182257 & 0.134380 & 0.424397 \\
3.0 & 0.116484 & 0.066361 & 0.050123 & 0.430298 \\
4.0 & 0.042852 & 0.024409 & 0.018444 & 0.430395 \\
5.0 & 0.015764 & 0.008979 & 0.006785 & 0.430396 \\
6.0 & 0.005799 & 0.003304 & 0.002496 & 0.430396 \\
7.0 & 0.0021335 & 0.001215 & 0.000918 & 0.430396 \\
8.0 & 0.00078486 & 0.000447 & 0.000338 & 0.430396 \\
9.0 & 0.000288735 & 0.0001645 & 0.000124 & 0.430389 \\
10.0 & 0.0001062198 & $6.053336 \times 10^{-5}$ & $4.568644 \times 10^{-5}$ & 0.430112 \\
\hline
\end{tabular}

Table 4: Absolute and relative errors obtained from UPFD scheme using $k=0.001$ with $h=0.1, a=1$, $D=1$ and $\kappa=1$.

Tables (3) and (4) compares the absolute error and relative error using two different values of $k ; 0.00025$ and 0.001 with $h=0.1$. The relative error is very small with $k=0.00025$ but it is too high (30 to 40 percent) when $k$ is chosen as 0.01 . This confirms that we must use a very small value of $k$ e.g. 0.00025 . For a scheme to be efficient, the relative error at a given value of $x$ must be less than 10 percent. 


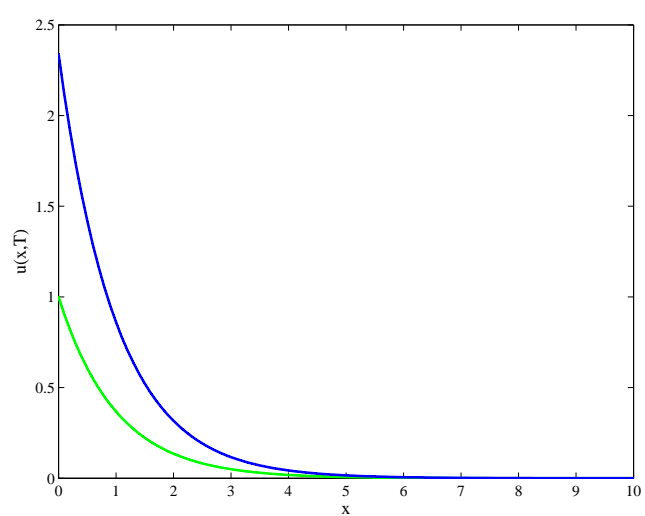

(a) $k=0.00025$

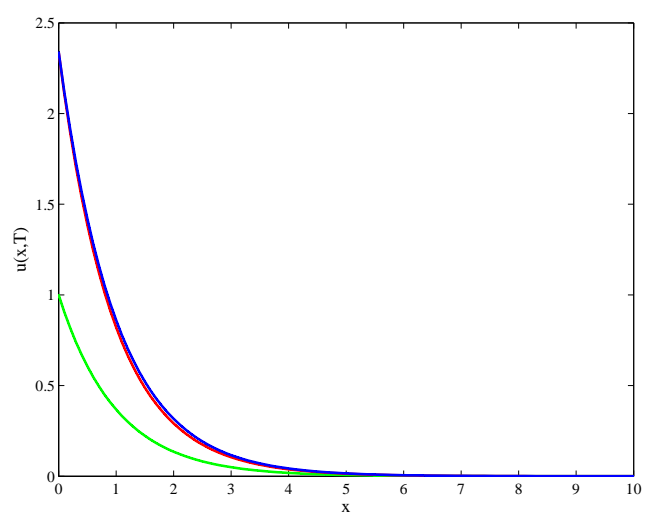

(b) $k=0.001$

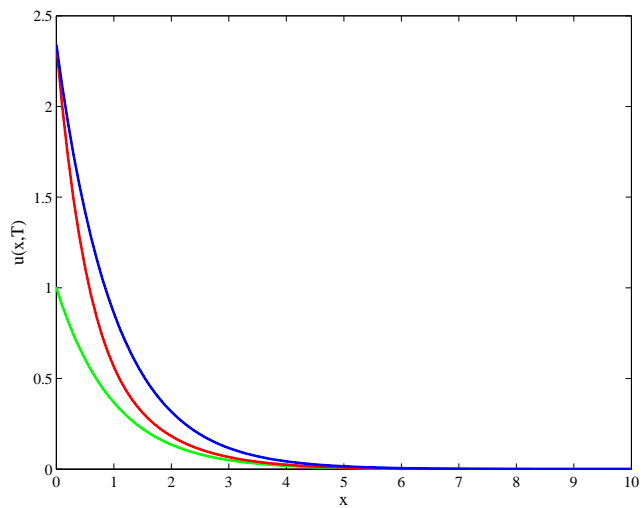

(c) $k=0.01$

Figure 1: Profiles of numerical solution (in red), exact solution (in blue) at time $t=0.85$ and initial solution (in green) using UPFD scheme at $a=1, D=1, \kappa=1$ and $h=0.1$. 


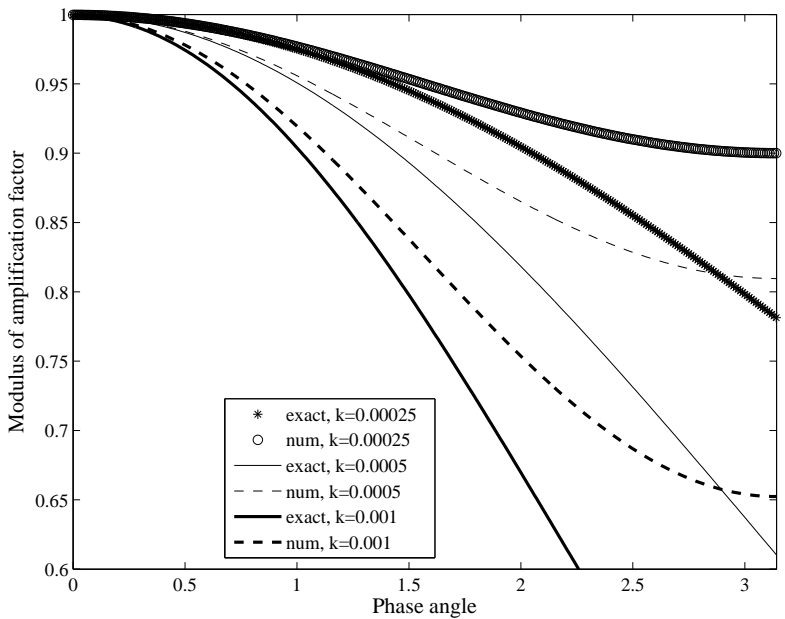

(a)

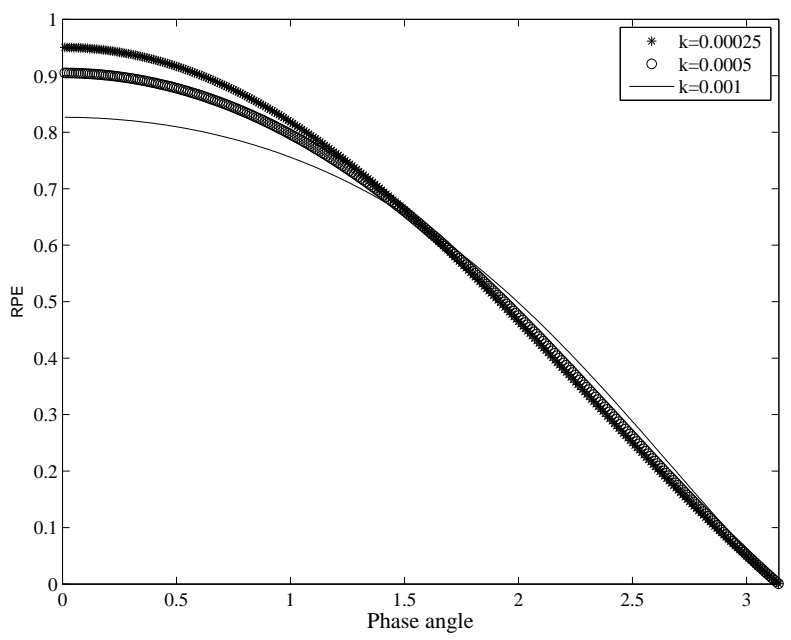

(c)

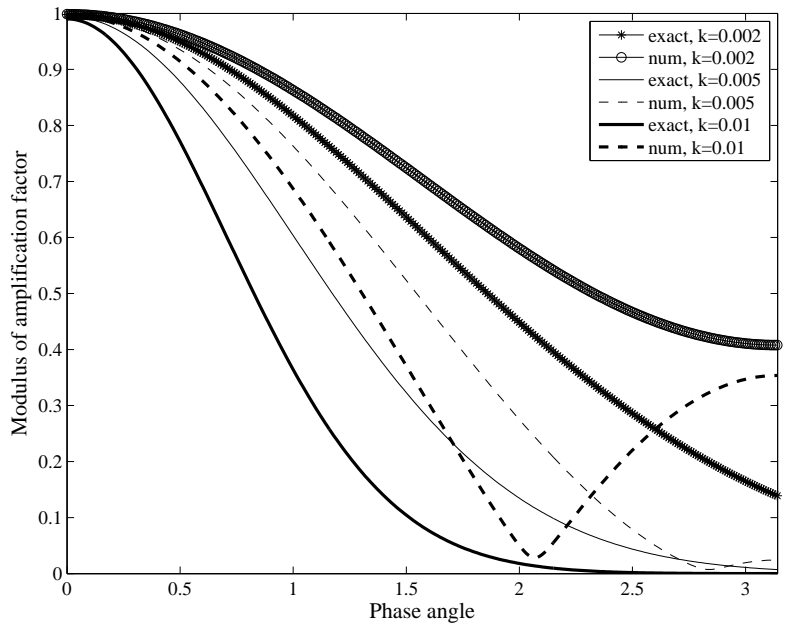

(b)

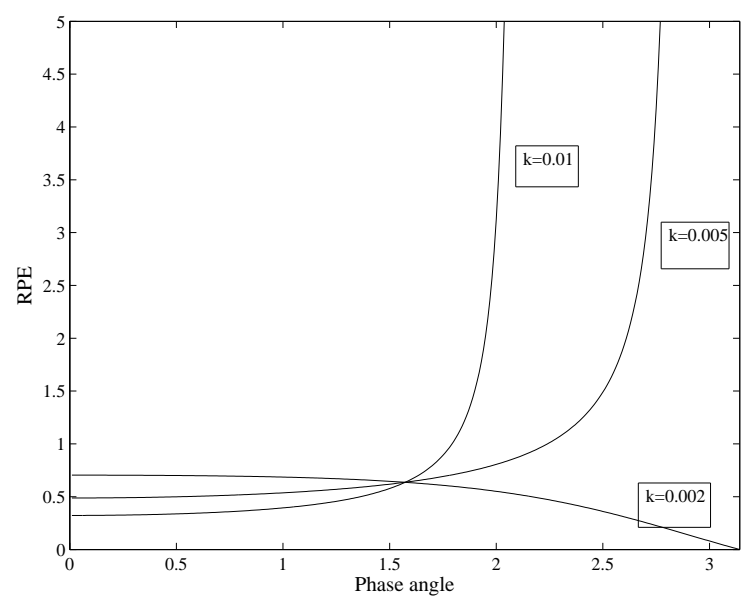

(d)

Figure 2: Plot of AFM and RPE vs phase angle for the UPFD scheme at some different values of $k$ with $h=0.1, a=1, D=1, \kappa=1$.

\section{Case 2: Advection is more important}

Errors such as $L_{1}$ error, dissipation and dispersion errors are displayed in Table (5) at some different values of $k ; 0.00025,0.0005,0.001,0.002,0.005,0.01$. The errors are larger compared to case 1 . We obtain satisfactory results at a small value of $k$ for instance at $k=0.00025$ but for other values of $k$, the errors are quite considerable. For instance at $k=0.00025$, we have the following errors; $L_{1}$ error $=0.5286$, Diss. error $=4.0980 \times 10^{-3}$, Disp. error $=2.9337 \times 10^{-3}$ while at $k=0.01$, the errors are; $L_{1}=39.9988$, Diss. error $=30.1971$, Disp. error $=26.3449$.

Table (6) compares relative errors at $k=0.00025$ and $k=0.0005$. The relative error is too large at $k=0.0005$. Hence, we can use very low value of $k$ i.e $k=0.00025$ for this numerical experiment when choosing $h=0.1$.

We can conclude that for $a=5, D=1, \kappa=1$, with $h=0.1$, UPFD only gives satisfactory results at values of $k$ very close to zero such as $k=0.00025$. Results deteriorate as $k$ is increased to 0.00050 


\begin{tabular}{|c|c|c|c|c|}
\hline$k$ & $L_{1}$ error & Diss. error & Disp. error & TMSE \\
\hline 0.00025 & 0.5286 & $4.0980 \times 10^{-3}$ & $2.9337 \times 10^{-3}$ & $7.0317 \times 10^{-3}$ \\
0.0005 & 3.2761 & 0.1596 & 0.1161 & 0.2757 \\
0.001 & 8.0751 & 0.9936 & 0.7437 & 1.7373 \\
0.002 & 15.6031 & 3.8620 & 3.01967 & 6.8879 \\
0.005 & 29.1988 & 14.7875 & 12.3561 & 27.1435 \\
0.01 & 39.9988 & 30.1971 & 26.3449 & 56.5421 \\
\hline
\end{tabular}

Table 5: Errors obtained from UPFD scheme at some different values of $k$ with $h=0.1, a=5, D=1$ and $\kappa=1$ at time $t=0.85$.

\begin{tabular}{|c|c|c|}
\hline$x$ & Relative error when $k=0.00025$ & Relative error when $k=0.0005$. \\
\hline 0 & 0 & 0 \\
1.0 & 0.007623 & 0.048209 \\
2.0 & 0.015184 & 0.094056 \\
3.0 & 0.022644 & 0.137346 \\
4.0 & 0.029809 & 0.176797 \\
5.0 & 0.036141 & 0.209171 \\
6.0 & 0.040852 & 0.230816 \\
7.0 & 0.043548 & 0.241545 \\
8.0 & 0.044658 & 0.245231 \\
9.0 & 0.044972 & 0.2460740 \\
10.0 & 0.044776 & 0.2459960 \\
\hline
\end{tabular}

Table 6: Relative errors obtained from UPFD scheme at 2 different values of $k: 0.00025$ and 0.0005 with $h=0.1, a=5, D=1$ and $\kappa=1$ at time $t=0.85$.

and larger values. Fig. (3(a)) and (3(b)) show numerical solution, exact solution at $k=0.00025$ and $k=0.001$. The deviation between numerical and exact solution is apparent even at a low value of $k$ such as 0.001 . We note that the peak value of numerical and exact solution at time, $t=0.85$ is approximately 70 though the peak value of initial solution is only one. This is explained by considering the exact value of $u$ at $x=0$. The value of $u(x=0, t=0.85)=\exp (4.25) \approx 70$ and this follows from Eq. (2).

Figs. (4(a)), (4(b)) depict plots of modulus of exact amplification factor, modulus of numerical amplification factor vs phase angle, at some different values of $k$ and we can observe that the curve for the numerical amplification factor is always above the exact amplification factor. Figs. (4(c)) and (4(d)) illustrate plots of RPE vs phase angle at different values of $k$ with $h=0.1$. For $k=0.00025,0.0005$, the scheme has quite good dispersive properties at low phase angles but as $k$ is increased from 0.0005 , the dispersive properties are not conserved even at low phase angles.

Table (7) shows relative error at $k=0.00025, h=0.1, a=10, D=1, \kappa=1$. The errors are in general greater than at $a=5$ and the results are quite satisfactory. For $a=100$, the errors are too considerable and UPFD scheme cannot be used in that case. 


\begin{tabular}{|c|c|}
\hline$x$ & Relative error when $k=0.00025$ \\
\hline 0 & 0 \\
1.0 & 0.018777 \\
2.0 & 0.037202 \\
3.0 & 0.055280 \\
4.0 & 0.0730192 \\
5.0 & 0.090424 \\
6.0 & 0.107487 \\
7.0 & 0.124129 \\
8.0 & 0.139997 \\
9.0 & 0.154180 \\
10.0 & 0.164272 \\
\hline
\end{tabular}

Table 7: Relative errors obtained from UPFD scheme using $k=0.00025, h=0.1, a=10, D=1$ and $\kappa=1$ at time $t=0.85$.

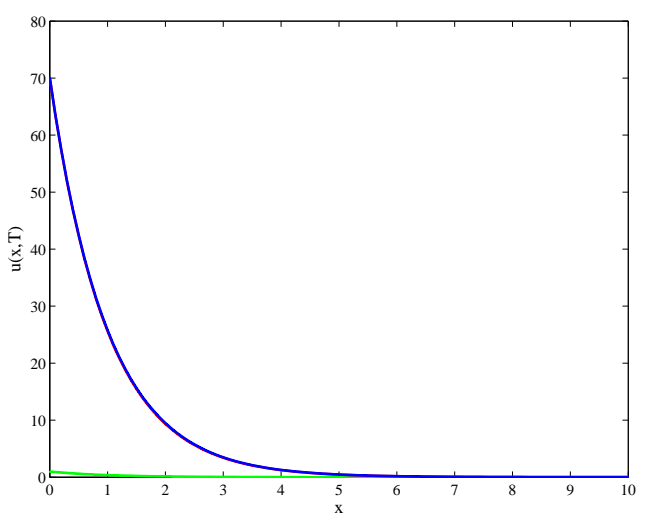

(a) $k=0.00025$

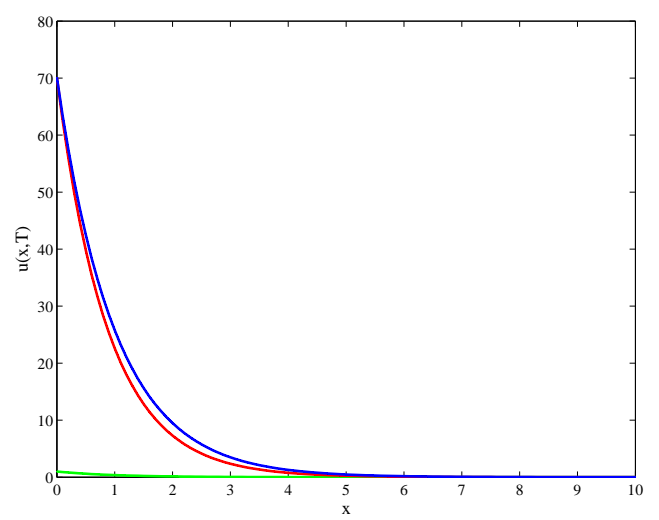

(b) $k=0.001$

Figure 3: Profiles of numerical solution (in red), exact solution (in blue) at time $t=0.85$, initial solution (in green) using UPFD at $a=5, D=1, \kappa=1$ with $h=0.1$. 


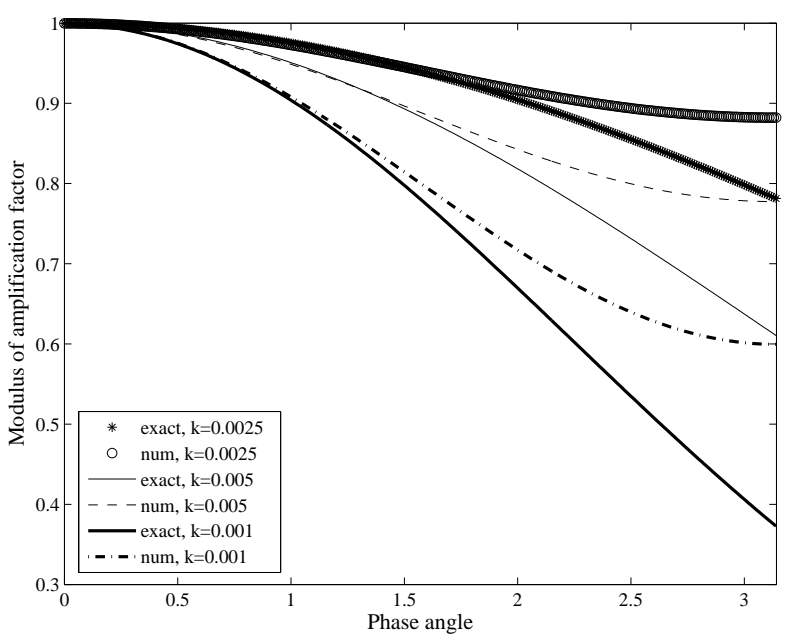

(a)

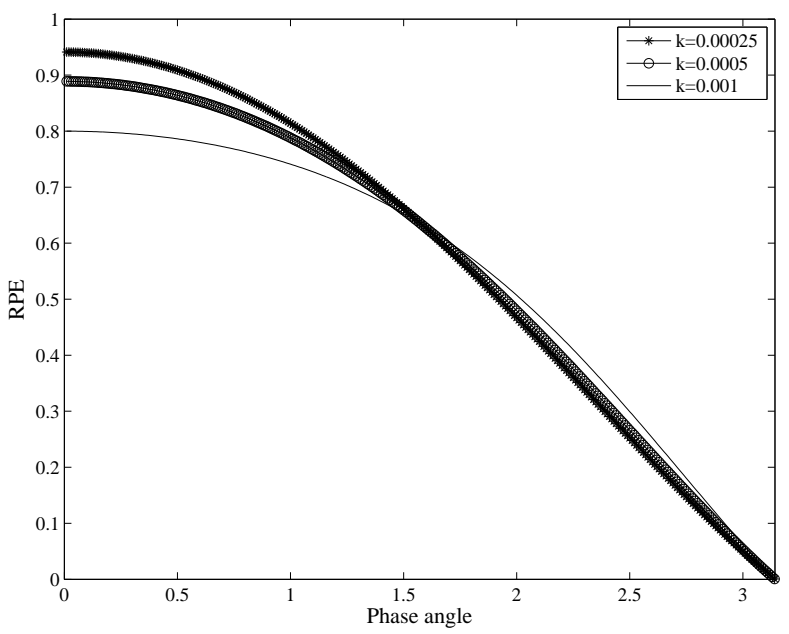

(c)

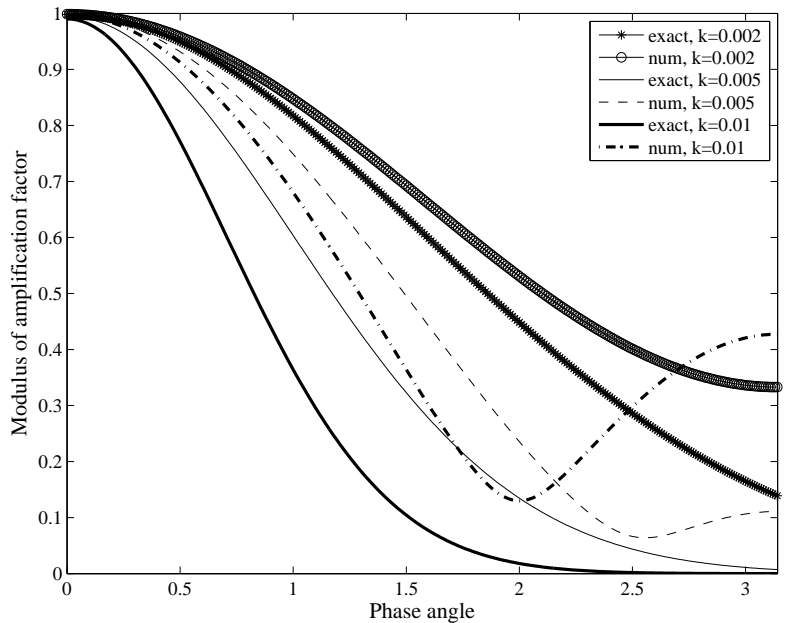

(b)

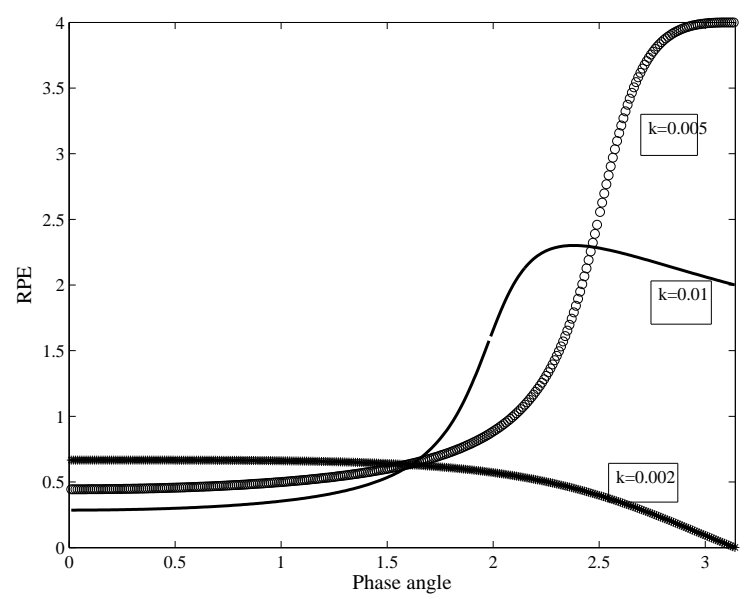

(d)

Figure 4: Plot of AFM and RPE vs phase angle for the UPFD scheme at $a=5, D=1, \kappa=1$ with $h=0.1$.

\section{Case 3: Diffusion is more important}

Table (3) shows $L_{1}$ errors, dissipation errors, dispersion errors at the values of $k ; 0.00025,0.0005,0.001$, $0.002,0.005,0.01$. The errors when compared at the same values of $k$ are larger compared to cases 1 and 2. At $k=0.00025$ we have $L_{1}=6.5711$, Diss. error $=0.6502$, Disp. error $=0.4792$ while at $k=0.0005$, we have $L_{1}=11.8129$, Diss. error $=2.1607$, Disp. error $=1.6411$. In general, the scheme cannot handle case 3. From Figs. (5(a)) and (5(b)), we observe that there is quite some deviation between numerical and exact profiles at $k=0.001$. We also note that like in case 2 , the peak value of the numerical and exact solution at time, $t=0.85$ is approximately 70 .

Plots of modulus of exact amplification factor and modulus of numerical amplification factor are displayed in Figs. (6(a)) and (6(b)) and we observe considerable deviation between these two profiles even at low phase angles and low values of $k$. The plots of numerical amplification factor are always above those for the exact amplification factor at a given value of $k$. Fig. (6(c)) shows plot of RPE 


\begin{tabular}{|c|c|c|c|c|}
\hline$k$ & $L_{1}$ error & Diss. error & Disp. error & TMSE \\
\hline 0.00025 & 6.5711 & 0.6502 & 0.4792 & 1.1294 \\
0.0005 & 11.8129 & 2.1607 & 1.6411 & 3.8018 \\
0.001 & 19.3297 & 6.0429 & 4.7828 & 10.8257 \\
0.002 & 28.4341 & 13.8789 & 11.499 & 25.4341 \\
0.005 & 40.4790 & 30.8990 & 26.9279 & 57.8270 \\
0.01 & 48.1032 & 46.8692 & 41.8260 & 88.6951 \\
\hline
\end{tabular}

Table 8: Errors obtained from UPFD scheme at some different values of $k$ with $h=0.1, a=1, D=5$ and $\kappa=1$.

\begin{tabular}{|c|c|c|c|c|}
\hline$x$ & Exact & Numerical & Absolute Error & Relative Error \\
\hline 0.0 & 70.105412 & 70.105412 & 0 & 0 \\
1.0 & 25.790340 & 23.244563 & 2.545777 & 0.098711 \\
2.0 & 9.487736 & 7.709446 & 1.778290 & 0.187430 \\
3.0 & 3.490343 & 2.559342 & 0.931001 & 0.266736 \\
4.0 & 1.284025 & 0.851606 & 0.432419 & 0.336768 \\
5.0 & 0.472367 & 0.284738 & 0.187629 & 0.397210 \\
6.0 & 0.173774 & 0.096016 & 0.077758 & 0.4474678 \\
7.0 & 0.0639279 & 0.0327898 & 0.0311381 & 0.4870812 \\
8.0 & 0.023518 & 0.011378 & 0.012140 & 0.516191 \\
9.0 & 0.0086517 & 0.0040173 & 0.00463443 & 0.5356673 \\
10.0 & 0.0031828 & 0.001450 & 0.0017328 & 0.5444251 \\
\hline
\end{tabular}

Table 9: Absolute and relative errors obtained from UPFD scheme using $k=0.00025$ with $h=0.1$, $a=1, D=5$ and $\kappa=1$.

vs phase angle at $k=0.00025,0.0005$ and 0.001 . The scheme has very bad dispersive properties at $k=0.001$ and satisfactory dispersive properties at $k=0.00025$. Table (9) displays the relative errors using $k=0.00025$ and $h=0.1$ and we conclude that the scheme cannot handle the case: $a=1, D=5$, $\kappa=1$ as the relative error varies from 0 to 54 percent.

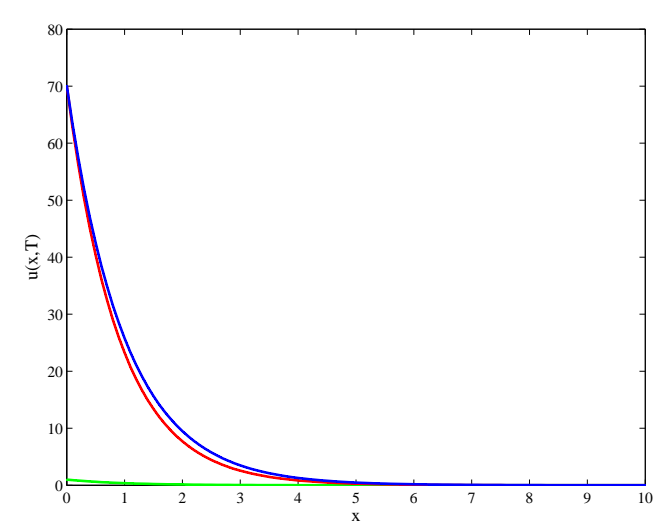

(a) $k=0.00025$

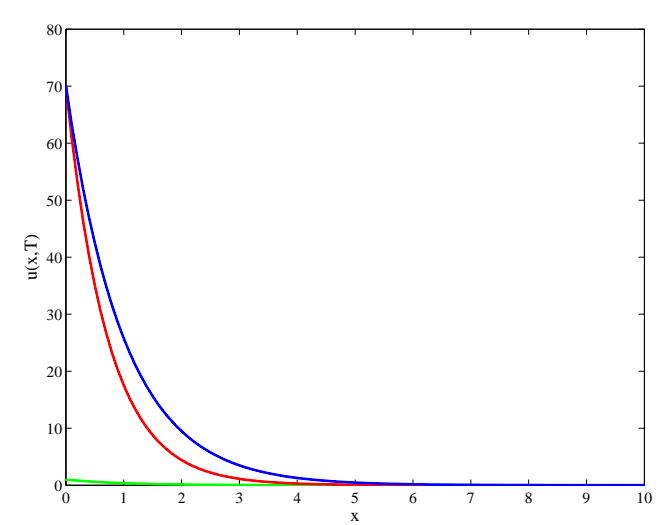

(b) $k=0.001$

Figure 5: Profiles of numerical solution (in red), exact solution (in blue) at time $t=0.85$ and initial solution (in green) using UPFD scheme at $a=1, D=1, \kappa=1$ with $h=0.1$. 


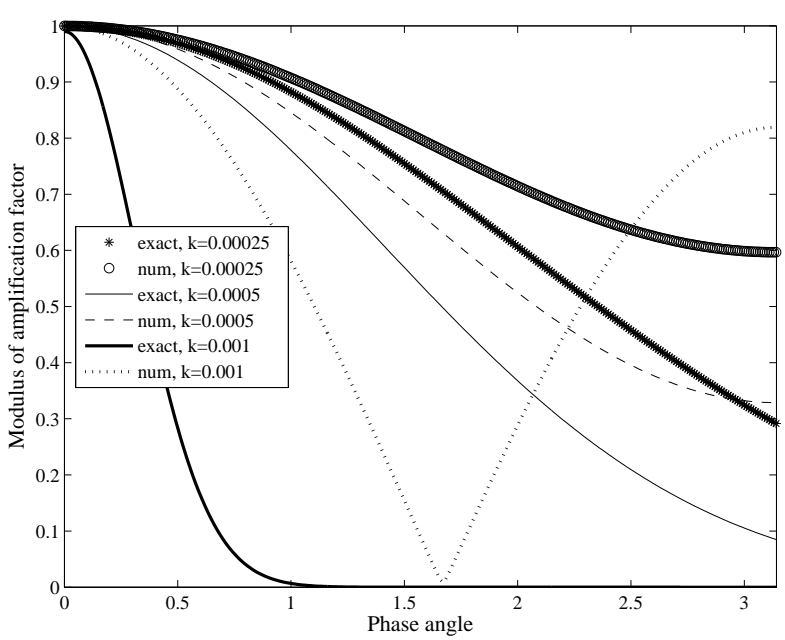

(a)

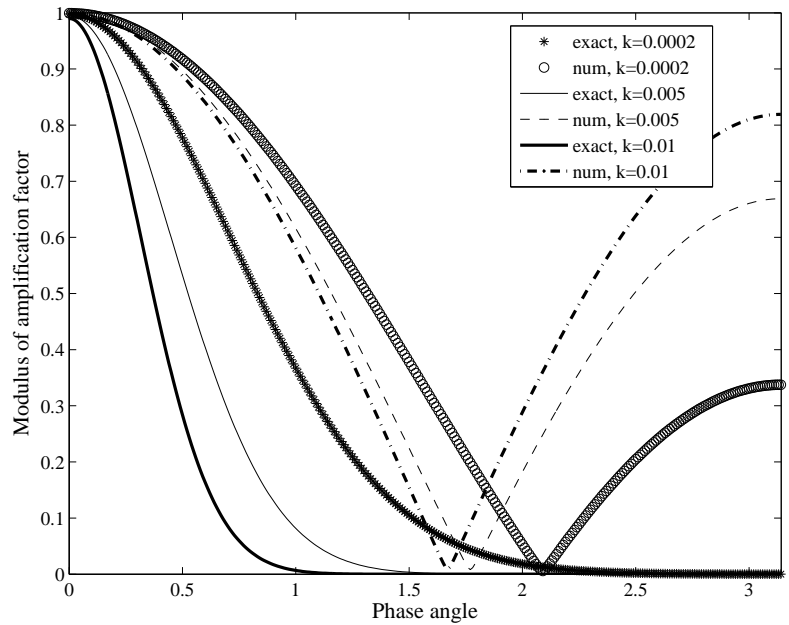

(b)

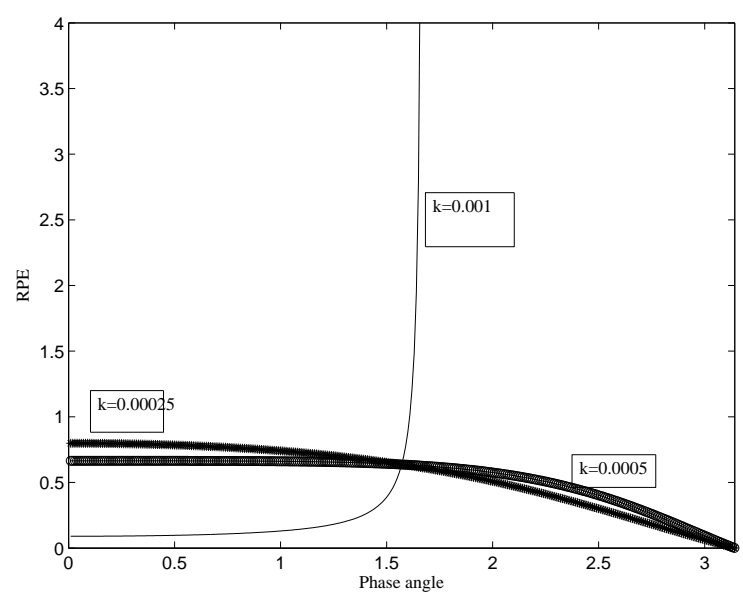

(c)

Figure 6: Plot of AFM and RPE vs phase angle for the UPFD scheme at $h=0.1, a=1, D=5$ and $\kappa=1$.

\section{Case 4: Reaction is more important}

Errors obtained at some different values of $k$ from UPFD scheme are tabulated in Table (4). In general, the UPFD scheme is satisfactory when reaction is more important and it works very well at low values of $k$ such as $0.00025,0.0005$. We display in Fig. $(7(\mathrm{a}))$ and $(7(\mathrm{~b}))$ the profiles for exact and numerical solution with $k=0.00025$ and 0.001 . We observe that there is very little deviation between the two plots at these two values of $k$. It is to be noted that the peak value of numerical and exact solution at $t=0.85$ is approximately 0.08. If we look at exact value of $u$ when $x=0$, we get $u(x=0, t=0.85)=\exp (-3 \times 0.85) \approx 0.08$.

Plots of AFM (exact and numerical) are displayed in Figs. (8(a)) and (8(b)) and we can observe that there is good agreement between exact and numerical AFM at very low values of $k$ and also at low phase angles. The dispersion character is quite good at low values of $k$ and especially at low phase angles. The scheme performs quite well at low values of $k$ as $\kappa \rightarrow \infty$ in constract to cases 2 and 3 . 


\begin{tabular}{|c|c|c|c|c|}
\hline$k$ & $L_{1}$ error & Diss. error & Disp. error & TMSE \\
\hline 0.00025 & $4.7650 \times 10^{-3}$ & $3.7488 \times 10^{-7}$ & $2.6310 \times 10^{-7}$ & $6.3798 \times 10^{-7}$ \\
0.0005 & $8.5700 \times 10^{-3}$ & $1.2273 \times 10^{-6}$ & $8.4216 \times 10^{-7}$ & $2.0694 \times 10^{-6}$ \\
0.001 & $1.6682 \times 10^{-2}$ & $4.7809 \times 10^{-6}$ & $3.1172 \times 10^{-6}$ & $7.8981 \times 10^{-6}$ \\
0.002 & $3.4527 \times 10^{-2}$ & $2.1838 \times 10^{-5}$ & $1.2610 \times 10^{-5}$ & $3.4448 \times 10^{-5}$ \\
0.005 & $9.4150 \times 10^{-2}$ & $1.9644 \times 10^{-4}$ & $7.5023 \times 10^{-5}$ & $2.7146 \times 10^{-4}$ \\
0.01 & $1.8956 \times 10^{-1}$ & $9.7191 \times 10^{-4}$ & $2.1300 \times 10^{-4}$ & $1.1849 \times 10^{-3}$ \\
\hline
\end{tabular}

Table 10: Errors obtained from UPFD scheme at some different values of $k$ with $h=0.1, a=1, D=1$ and $\kappa=5$ at time, $t=0.85$.

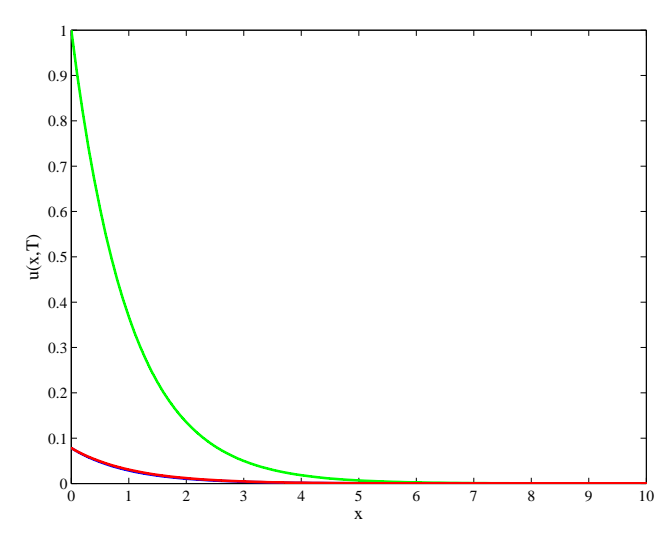

(a) $k=0.00025$

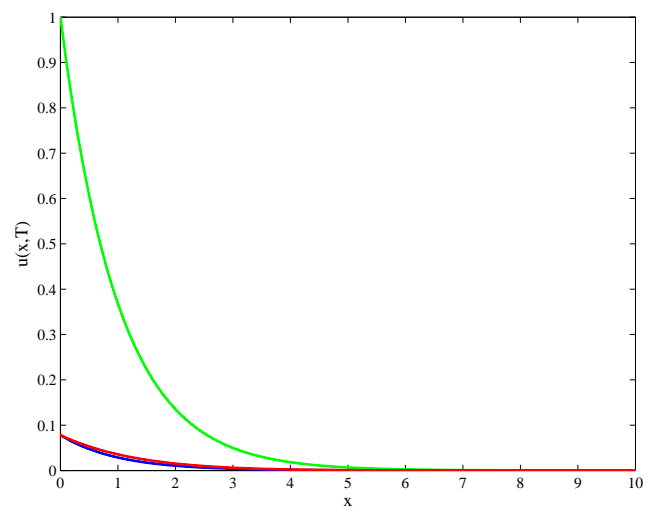

(b) $k=0.001$

Figure 7: Profiles of numerical solution (in red), exact solution (in blue) at time, $t=0.85$ and initial solution (in green) using UPFD scheme at $a=1, D=1, \kappa=5$ with $h=0.1$. 


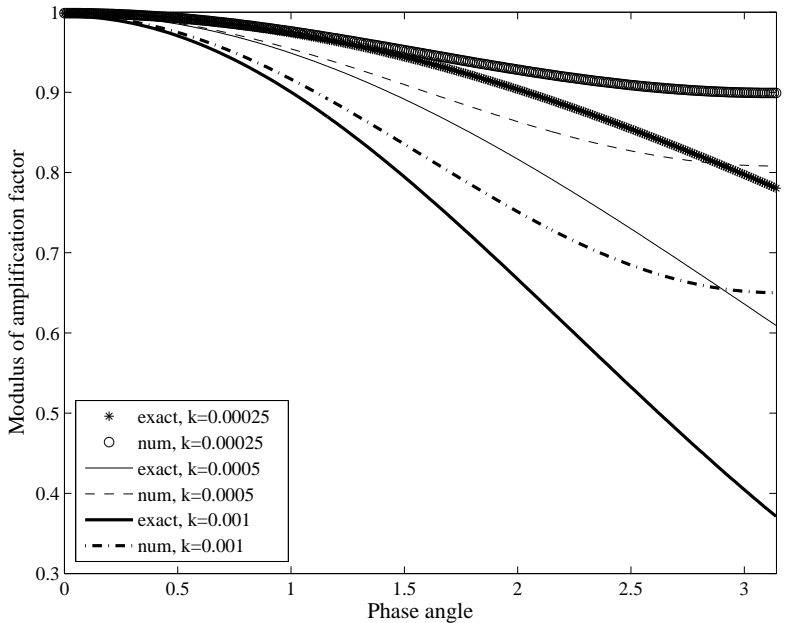

(a)

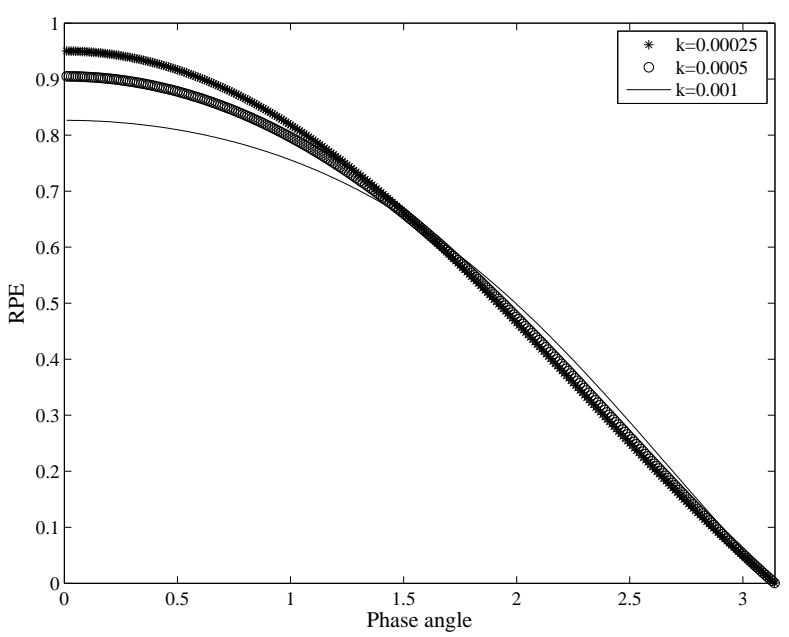

(c)

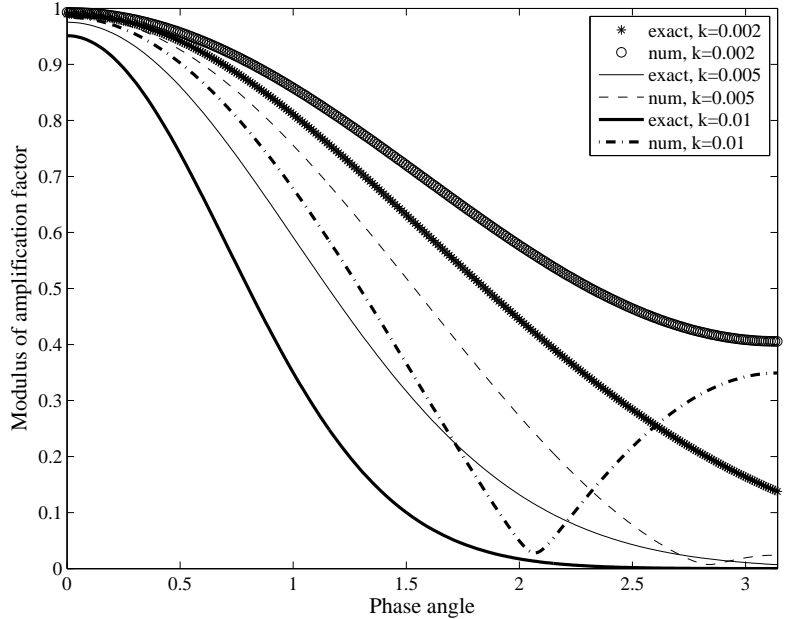

(b)

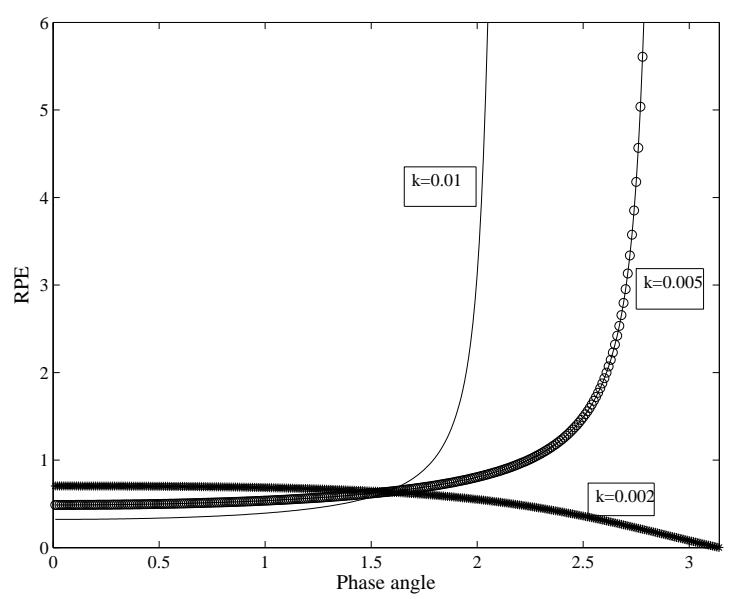

(d)

Figure 8: Plots of AFM and RPE vs phase angle for the UPFD scheme at $a=1, D=1, \kappa=5$ with $h=0.1$.

\section{Conclusion}

The UPFD scheme is unconditionally stable but not unconditionally consistent. The scheme was tested on an advection-diffusion-reaction equation which models exponential travelling waves and we computed various errors such as $L_{1}$ error, dispersion and dissipation errors, relative errors under some different regimes of advection, diffusion and reaction. The scheme works best for very small values of $k$ as $k \rightarrow 0$, (for instance, $k=0.00025,0.0005$ ) and performs satisfactorily at other values of $k$ such as 0.001 for two regimes; $a=1, D=1, \kappa=1$ and $a=1, D=1, \kappa=5$. When $a=5, D=1, \kappa=1$, the scheme performs quite well at $k=0.00025$ and satisfactorily at $k=0.0005$ but is not efficient at larger values of $k$. For the diffusive case $(a=1, D=5, \kappa=1)$, the scheme does not perform well. In general, we can conclude that the choice of $k$ is very important as it affects to a great extent the performance of the method. The UPFD scheme is effective to solve advection-diffusion-reaction problems when advection or reactive regime is dominant and for the case, $a=1, D=1, \kappa=1$ especially at low values of $k$. Moreover, the 
magnitude of the dispersion and dissipation errors using UPFD are of the same order for all the different regimes considered as seen from the tables. This indicates that if we are to optimise the temporal step size at a given value of the spatial step size, our optimisation function must consist of both the $A F M$ and RPE. Some related work on optimisation can be seen in [2]. Higher order unconditionally stable schemes can be constructed for the regimes for which UPFD is not efficient enough for instance when advection and diffusion are dominant.

\section{Acknowledgements}

A short version of this paper was presented at the $10^{\text {th }}$ International Symposium on Numerical Analysis of Fluid Flow and Heat Transfer, Numerical Fluids 2015 held in Rhodes, Greece from 23 to 29 September 2015.

\section{Nomenclature}

$\alpha$ : Dispersion relation

$I: \sqrt{-1}$

$k$ : Time step

$h$ : Spatial step

$\theta$ : Wave number

$w$ : Phase angle

$\xi_{\text {exact }}$ : Exact amplification factor

$\xi_{\text {num }}$ : amplification factor of numerical method

AFM: Amplification Factor Modulus (Modulus of Amplification Factor)

RPE: Relative phase error

Diss. error: Dissipation error

Disp. error: Dispersion error
$a$ : advective velocity
$D$ : Diffusion coefficient
$n$ : Time level
$i$ : Mesh point location in $x$-direction

\section{References}

[1] O. Abu Arqub and A. El-Ajou, S. Momani, Constructing and predicting solitary pattern solutions for nonlinear time-fractional dispersive partial differential equations, Journal of Computational Physics 293, 385-399, 2015.

[2] A. R. Appadu, Numerical solution of the 1-D advection-diffusion equation using standard and nonstandard finite difference schemes, Journal of Applied Mathematics 15, 201-215, 2013. 
[3] A. R. Appadu and M. Z. Dauhoo, The concept of minimized integrated exponential error for low dispersion and low dissipation, International Journal for Numerical Methods in Fluids, 65, 578-601, 2011.

[4] A.R. Appadu, J.M.S. Lubuma and N. Mphephu, Computational study of three numerical methods for some linear and non-linear advection-diffusion-reaction problems, Progress in Computational Fluid Dynamics (In Press).

[5] B. M. Chen-Charpentier and H. V. Kojouharov, Non-standard numerical methods applied to subsurface biobarrier formation models in porous media, Bulletin of Mathematical Biology 61, 4, 779-798, 1999.

[6] B.M.Chen-Charpentier and Hristo V. Kojouharov, An unconditionally positivity preserving scheme for advection-diffusion-reaction equation, Mathematical and Computer Modelling, 57, 2177-2185, 2013.

[7] R.M.Clancy, A note on finite differencing of the advection-diffusion equation, Monthly weather Review, 109, 1807-1809, 1981.

[8] www.mathematik.uni-dortmund.de/ kuzmin/cfdintro/lecture9.pdf

[9] A. El-Ajou, O. Abu Arqub and S. Momani, Approximate analytical solution of the nonlinear fractional kdV-Burgers equation a new iterative algorithm, Journal of Computational Physics, 293, 2015, 81-95.

[10] A. El-Ajou, O. Abu Arqub, S. Momani, D. Baleanu and A. Alsaedi, A novel expansive iterative method for solving linear partial differential equations of fractional order, Applied Mathematics and Computation, 257, 2015, 119-133.

[11] J. Fromm, The time dependent flow of an incompressible viscous fluid, Methods in Computational Physics, 3, 345-382, 1964.

[12] A.C. Hindmarsch, P.M. Gresho, D.F. Griffiths. The stability of explicit Euler time-integration for certain finite difference approximations of the multi-dimensional advection-diffusion equation, International Journal for Numerical Methods in Fluids, 4, 853-897, 1984.

[13] C. Hirsch, Numerical computation of internal and external flows, Vol. 2, John Wiley and Sons, 1990.

[14] C.W.Hirt, Heuristic stability theory for finite difference equations, Journal of Computational Physics, 2, 339-355, 1968.

[15] A. Rigal, Stability analysis of explicit finite difference schemes for the Navier-Stokes equations, International Journal of Numerical Methods in Engineering, 14, 617-620, 1979.

[16] Tony W.H. Sheu, S.K. Wang and R.K. Lin, An implicit scheme for solving the convection-diffusionreaction equation in two dimensions, Journal of Computational Physics, 164, 123-142, 2000.

[17] J. Siemieniuch and I. Gladwell, Analysis of explicit difference methods for the diffusion-convection equation, International Journal of Numerical Methods in Engineering, 12, 899-916, 1978.

[18] E. Sousa, The Controversial Stability Analysis, Applied Mathematics and Computation, 145, 777794, 2003.

[19] L. Takacs, A two-step scheme for the advection equation with minimized dissipation and dispersion errors, Monthly Weather Review, 113, 1050-1065, 1985. 
[20] J.C. Tannehill, D.A. Anderson and R.H. Pletcher, Computational Fluid Mechanics and Heat Transfer, $2^{\text {nd }}$ Edition, Taylor and Francis, 1997.

[21] Hy Trac and Ue-Li Pen, A primer on eulerian computational fluid dynamics for astrophysics, Astronomical society of the pacific, 115, 303-321, 2003.

[22] K.W. Morton and D.F. Mayers, Numerical solution of partial differential equations, Cambridge University Press, 1994. 